\title{
Publication, évaluation : sortons du diktat du facteur d'impact !
}

Publier est une étape incontournable de la production des connaissances et du métier de physicien. La communauté scientifique et l'édition scientifique partagent l'exigence de qualité de cette construction des connaissances, organisée autour de la vérification et de la validation par les pairs. Malheureusement, certains pans de la vie de l'édition scientifique ne procèdent pas seulement de cette convergence. Les nouvelles composantes marchandes de cette chaîne d'activité rongent, dissolvent, d'une façon presque indolore les valeurs de l'éthique scientifique, et ouvrent des fenêtres à la fraude.

Dans le numéro 43 de Reflets de la physique, l'article de M. Knoop et al. présente une analyse des publications de I'Institut de physique du CNRS. Sur cinq ans (2007-2011), seuls $10 \%$ des articles publiés ont un facteur d'impact supérieur à 7 . Les cinq revues « prestigieuses » multidisciplinaires cumulées contribuent à 2,5\% seulement du total. Cette frange de l'édition, et pas uniquement elle, réunit la présentation d'excellents résultats qui construisent leur prestige mais aussi, trop fréquemment, introduisent des pratiques aux antipodes de l'éthique scientifique. Ces dérives ne peuvent pas être ignorées par notre communauté. À terme, l'érosion des valeurs de la démarche scientifique au sein de l'édition serait un désastre irréversible pour l'ensemble de la recherche.

Physiciens, créateurs de connaissances scientifiques ${ }^{(1)}$, formateurs de docteurs, mais également pairs en charge d'évaluation, membres de jurys de recrutement ou de prix, nous devons refuser le diktat du facteur d'impact qui érode l'excellence, plus exigeante, plus subtile à concevoir.

- La machine. Quelques éditeurs, pas uniquement ceux producteurs de journaux à haut facteur d'impact, animés principalement par leur démarche marchande, hyperrentable actuellement, deviennent les maîtres quasi exclusifs de la machinerie, profitant d'un rapport de force pour imposer des règles nouvelles au publiant et des abonnements en bouquet au lecteur et ses bibliothèques. Ces «lois» sont incompatibles avec le développement et la propriété des biens culturels et scientifiques, biens communs forgés pour leur majeure partie par les contribuables de tous les pays ${ }^{(2)}$

- Le carburant. Il alimente la machine et est fourni par nous, scientifiques. Nous nourrissons cette évolution pernicieuse, à la fois comme créateurs de la connaissance et comme multi-évaluateurs en tant que pairs. Surchargé et pressé, le scientifique est poussé à s'aligner sur le facteur d'impact dans l'appréciation de l'excellence.

Est-ce une descente inéluctable ? Fort heureusement, certaines communautés échappent à cette spirale destructrice. Les mathématiciens dans toute leur diversité, les physiciens des hautes énergies autour du CERN et SCOAP3, se sont organisés pour échapper aux diktats des majeurs de l'édition scientifique.

Les fortes évolutions de ces dix dernières années contribuent à cette érosion.

- Multiplication et qualité de l'évaluation sont antinomiques. La segmentation excessive des sources de financement de la recherche, le développement important des comités de toutes catégories, multiplient le travail d'expertise et conduisent à une évaluation de plus en plus hâtive, basée sur des indices de toutes natures (facteur d'impact compris), au détriment d'une appréciation qualitative, collective, issue d'une lecture intelligente des articles.

- La révolution numérique. Une grande chance pour la diffusion et le partage des connaissances nouvelles, elle facilite l'accès rapide aux résultats de la recherche. Mais elle permet aussi à quelques gros éditeurs d'accroître considérablement leur pouvoir, forgeant de nouvelles pratiques éloignées de l'éthique de la publication scientifique. Les éditeurs adossés aux sociétés savantes, telle que la SFP, plus soucieux de l'éthique, en sont gravement fragilisés.

Freiner et infléchir radicalement cette dérive est aussi la responsabilité des physiciens. Qu'ils soient créateurs de connaissances, formateurs de doctorants, membres de jurys, université et organisme, ou Académie des sciences, résister, aujourd'hui, aux appels miroitants des gros éditeurs est essentiel.

Alain Fontaine

Président de la Société Française de Physique 